\title{
A Prospective Study on Endoscopic Versus Surgical Management of Choledocholithiasis
}

\author{
${ }^{1 *}$ Dr.P.V.Durga Rani, ${ }^{2}$ Dr.Chanda Ramana Chalam, Dr. T Prasad, \\ ${ }^{4}$ Dr. K.Appa Rao. \\ ${ }^{1,2}$ Assistant Professor, ${ }^{3}$ Post Graduate, ${ }^{4}$ Professor : Department Of General Surgery, Siddhartha Medical \\ College, Vijayawada, Andhra Pradesh.
}

\begin{abstract}
Introduction: Choledocholithiasis is the most common cause of obstructive jaundice and occurs in about 10\% of patients with symptomatic gallstone. The need for subsequent cholecystectomy in patients with gall bladder in situ after endoscopic removal of stones from the common bile duct is controversial.

Methodology: A prospective study was conducted from November 2014 to march 2016 in patients diagnosed to have Choledocholithiasis . Patients with intra hepatic stones and biliary stricture were excluded from the study. A total of 35 with a diagnosis of Choledocholithiasis were included in this study.

Results: There was a slightly increased incidence in male patients (M:F 1:0.94). Pain abdomen, jaundice and fever are the common clinical symptom, Serum bilirubin and alkaline phosphates levels were usually deranged. Ultrasonography has $70.9 \%$ sensitivity. MRCP and CT done only in patients ultrasonography was negative. ERCP with ES had a success rate of 86.36\%. Cholecystectomy was done in 9(4.36\%) patients after ERCP. 12(38.7\%) patients underwent open surgical procedures. Escherichia coli and klebsialla spp the most common organisms isolated. Complications were more common in patients whom underwent open surgery. Mean hospital stay in endoscopic interventions was 11.84 days. Patients who underwent open surgery had more prolonged hospital stay with a mean of 26-67 days.

Conclusion: Ultrasonography has a sensitivity of $70.96 \%$ in detecting calculi. Routine se of MRCP and CT is not required. ERCP with ES can be used as the only intervention for the treatment of CBD calculi in patients with a normal gall bladder. Cholecystectomy needs to be done only in patients with gall bladder pathology. Complications are more in patients whom undergo open surgery. There is a significant decreased mean hospital stay in patients who undergo endoscopic treatment.
\end{abstract}

Keywords: Choledocholithiasis, ERCP, MRCP, Sphincterotomy, cholecystectomy

\section{Introduction}

Langenbuch performed the first cholecystectomy in 1882. It was not until several years later that Robert Abbe performed the first cholecystectomy. Soon enough, choledochotomy became the golden standard notwithstanding the fact that addition of the ductal exploration to cholecystectomy raises the morbidity and mortality significantly. ${ }^{3,4}$ With the gaining experience, these rate started falling; nevertheless there was a definite risk. This is related to the degree of obstructive jaundice and the presence of medical risk factors. Biliary obstruction, which is the interruption from the liver to small intestine, can occur at any level with in biliary system. Choledocholithiasis is most commonly a complication of gall bladder stones; only in minority of patients they arise de novo in the bile ducts. Its incidence in the developing nations is continuously rising. ${ }^{1,2}$ Though they are seen in all age groups, it is the 'fat, fertile', flatulent, female of fifty' that is most commonly affected. The incidence increases with age. ${ }^{3}$ the incidence also been rising this part of the country; possible reasons are the changing dietary habits, increasing awareness of health in people and improvements in the imaging technology.

A patient with ductile stones was at the mercy of the natural course of the disease until the late $19^{\text {th }}$ century; fortunate, if there was spontaneous passage of the stone, or eventually succumb to the complications Many studies have proven that pre operative endoscopic Sphincterotomy and surgery offers no advantage over surgery alone. ${ }^{5}$ However, endoscopy is a safe option in elderly patients and in those with medical risk factors. It relives obstruction, improves the general condition in the settling of acute cholangitis with a much lower mortality. Newer endoscopic techniques have claimed a good clearance rate for the large stones too. Laparoscopic cholecystectomy has been extensively accepted since Mouret first successfully finished the procedure in $1977^{6}$. The advent of laparoscopy dramatically changed the scenario. Management of Choledocholithiasis has become even more controversial. Though some centers have specialized in laparoscopic common bile duct exploration, many resort to preoperative Sphincterotomy for ductal stones. 
The aims of the present study were to analyze patients with Choledocholithiasis and study demographic profile, etiological, pre disposing factors, spectrum of clinical presentation, alterations in liver function tests, bacteriological characteristics, sensitivity of investigative modalities like ultrasonography, CT and MRCP and to outline the strategies for management and hence the best mode of management of the calculus.

\section{Methodology}

A prospective study was conducted from November 2014 to march 2016 in patients diagnosed have Choledocholithiasis in. They were followed up for a period of 6 months. Patients with intra-hepatic stones and biliary stricture were excluded from the study. A total of 35 patients with a diagnosis of Choledocholithiasis are included in this study. Detailed history was elicited including operative risk factors, previous biliary surgeries and a thorough physical examination was done. History regarding the age, jaundice, pain, fever, nausea/vomiting, pale stools, pruritis, cholangitis and pancreatitis was recorded. Medical risk factors such as diabetes, hypertension, ischemic heart diseases etc were considered.

Yellowish discoloration of sclera was considered as jaundice. Pain was considered biliary origin if it was located over the right upper abdomen with or without radiation to lower right scapula. Fever was either subjective where the patient described it or objective if the recorded temperature was more than $98.6^{0} \mathrm{~F}$ with or without chills and rigors. Nausea and vomiting if occurred during the episode of pain was considered to be of biliary cause. Stools without the normal yellowish or brownish color where considered as pale stools. Itching is not attributable to any dermatologic or systemic condition was considered as pruritis due to biliary pathology. Pancreatitis was defined as acute onset of epigatric pain with or without radiation to back and raised serum analysis levels. Patients who had previous biliary surgery and presented with CBD calculi was considered to be with retained stones if the stone developed within 2 years from the date of previous surgery and recurrence stones if it developed more than 2 years. Diabetic patients were those who required long-term oral hypoglycemics/insulin or those who were diagnosed upon admission. Similarly hypertensives were those who were on anti hypertensive drugs or diagnosed upon admission.

Biochemical investigations including random blood sugar, urea, creatinine, liver function tests were done in all patients. Serum bilirubin was raised if it was more than $0.8 \mathrm{mg} / \mathrm{dL}$. Alkaline phosphatase was raised if it was more than $115 \mathrm{u} / \mathrm{L}$. Ultrasonography was performed in all patients with a clinical suspicion of CBD calculi. Dilatation of intra and extra hepatic biliary radicals was considered as an indirect evidence of CBD stones. Presence or absence of gall bladder stones or wall thickened was also noted. CT scan with MRCP was performed in patients in whom ultrasonography was not able to pick up stones in the CBD detect dilatation of the biliary radicals.

Stones were considered if the stones if they were found in CBD stones or considered secondary if they also have gall bladder stones. ERCP was done in 23 patients. If stones were demonstrated, an endoscopic sphincterotomy was done in all patients and their retrieval was attempted. Stones were retrieved by either basketing or with balloon which was left to the discretion of the endoscopist. A $10 \mathrm{~cm}$ IOF CBD stent was inserted in all patients, which was to be removed after 6 weeks.

ERCP was considered as a sole procedure in patients with only CBD stones and with a normal gall bladder. It was followed by cholecystectomy in patients with either gall stones or cholecystitis.

Laparoscopic cholecystectomy was attempted in all patients, failure of which entailed open cholecystectomy. For the sake of analysis these patients are grouped under endoscopy.

Inclusion criteria : Old patients age > $60 \mathrm{yrs}$, Primary CBD stones, Patients with co-morbid conditions and with acute cholangitis

Patients with stone size $>1 \mathrm{~cm}$ and patients in whom ERCP was a failure were subjected to open surgery.

\section{The procedures performed were:}

1. CBD exploration

2. CBD exploration with transduodenal Sphincterotomy

3. CBD exploration with choledocho-jejunostomy.

Cholecystectomy was done in all patients who underwent open surgery.

Patients underwent CBD exploration if:

1. Large stone were detected at ultrasound, CT or MRCP

2. They were young and fit

3. ERCP/ES had failed

Abdomen was opened with a right subcostal or right paramedian incision. Common duct was considered dilated if it was more than $0.9 \mathrm{~cm}$. After Kocher's manoeuvre and skeletonization of the duct, CBD was palpated from above downwards. A L5 to $2.5 \mathrm{~cm}$ vertical incision was made on the CBD and bile sent for 
culture sensitivity. All stones were extracted. Baker's dilators were used to clear the duct of sludge and small stones. Duct was flushed with saline and 10 to $14 \mathrm{~F}$ T-tube placed in choledochotomy opening and duct snugly sutured around the tube with absorbable sutures. T-tube was removed after a period of 10 days if the t-tube cholangiogram showed good flow of the dye into the duodenum.

Perioperative cholangiogram was used selectively, the indications being, Sonologically proven stones that could not be located preoperatively, If the clearance of duct was doubtful after the retrieval of stones. One patient underwent choledochojejunostorny. Massively dilated CBD $(3.5 \mathrm{~cm})$ with narrowing of the distal CBD with multiple impacted stones was the indication. Anastomosis was done in a single layer with interrupted absorbable sutures and a drain placed in the vicinity of the anastomotic site. Transduodenal sphincteroplasty was done in one patient with an impacted stone at the ampulla of Vater. Patients, after surgery were monitored for postoperative complications. Hospital stay was recorded in all .cases. Wound infection was defined as the absence of primary healing with or without positive bacterial culture, requiring antibiotics with or without drainage. Chest infection was defined as production of purulent sputum or by its demonstration in chest x-ray, and requiring antibiotics and chest physiotherapy. Patients were observed for abdominal complication including bile leaks from drain site and intra- abdominal collections. The patients were divided into 2 groups - surgery and endoscopy for determining complication rates. The difference in the mean duration of hospital stay in each group was also checked for significance.

\section{Statistical methods:}

Descriptive statistical analysis has been carried out in the present study. Results on continuous measurements are presented on Mean+_SD (Min-Max) and results on categorical measurements are presented in Number (\%). Significance is assessed at $5 \%$ level or significance. The following assumptions on data are made, Assumptions: 1. Dependent variables should be normally distributed, 2.Samples drawn from the population should be random, and Cases of the samples should be independent.

Chi- square/Fisher Exact test has been used to find the significance of study parameters on categorical scale between two or more groups.

\section{Results}

Thirty five patients were included in this study. Seventeen patients $(48.5 \%)$ were females and $18(51.5 \%)$ were males, thus the female to male ratio is $0.94: 1$.

\begin{tabular}{|c|c|c|c|c|c|c|c|}
\hline \multirow[t]{2}{*}{ S.NO } & \multirow{2}{*}{$\begin{array}{l}\text { Age in } \\
\text { years }\end{array}$} & \multicolumn{2}{|c|}{ ERCP+ } & \multicolumn{2}{|c|}{ Open surgery } & \multicolumn{2}{|c|}{ All patients } \\
\hline & & No & $\%$ & No & $\%$ & No & $\%$ \\
\hline 1 & $30-40$ & 4 & 17.4 & 2 & 16.7 & 6 & 17.1 \\
\hline 2 & $41-50$ & 7 & 30.4 & 2 & 16.7 & 9 & 25.7 \\
\hline 3 & $51-60$ & 5 & 21.7 & 2 & 16.7 & 7 & 20 \\
\hline 4 & $61-70$ & 6 & 26.1 & 4 & 33.3 & 10 & 28.6 \\
\hline 5 & $>70$ & 1 & 4.3 & 2 & 16.7 & 3 & 8.6 \\
\hline 6 & Total & 23 & 100 & 12 & 100 & 35 & 100 \\
\hline 7 & $\begin{array}{l}\text { Mean } \\
+ \text { +SD }\end{array}$ & \multicolumn{2}{|c|}{$53.84+114.25$} & \multicolumn{2}{|c|}{$59.25+\_13.91$} & \multicolumn{2}{|c|}{$55.94+\_14.14$} \\
\hline
\end{tabular}

\section{Table no 1: Age distribution}

The age at presentation in the females ranged from 30 to 75 years with a mean age of 55.75 years (SD 13.56). Overall the mean age of presentation was 55.75 years (SD 14.64). Thus, most of the females presented at a late age than males. Age distribution is stastically similar in both groups with $\mathrm{p}=0.317$. (Table 1 )

\begin{tabular}{|c|c|c|c|c|c|}
\hline S.NO & Clinical symptoms & $\begin{array}{l}\text { ERCP+ } \\
\mathrm{n}=23\end{array}$ & Open surgery $n=12$ & All patients $n=35$ & $\mathrm{P}$ value \\
\hline 1 & Icterus & $11(47.8 \%)$ & $8(66.7 \%)$ & $18(51.4 \%)$ & 0.701 \\
\hline 2 & Pain & $20(87 \%)$ & $12(100 \%)$ & $32(91.4 \%)$ & 1.000 \\
\hline 3 & Fever & $10(43.5 \%)$ & $4(33.3 \%)$ & $14(40 \%)$ & 0.234 \\
\hline 4 & Pancreatitis & $3(13 \%)$ & $1(8.3 \%)$ & $4(11.4 \%)$ & 0.515 \\
\hline 5 & Pale stools & $2(8.7 \%)$ & $3(25 \%)$ & $5(14.2 \%)$ & 0.545 \\
\hline 6 & Pruritis & $2(8.7 \%)$ & $1(8.3 \%)$ & $3(8.6 \%)$ & 1.000 \\
\hline 7 & Chills & $7(30.4 \%)$ & $4(33.3 \%)$ & $11(31.4 \%)$ & 1.000 \\
\hline 8 & Nausea/vomiting & $16(69.5 \%)$ & $5(41.7 \%)$ & $21(60 \%)$ & $0.031^{*}$ \\
\hline 9 & $\mathrm{H} / \mathrm{O}$ jaundice & $12(52.1 \%)$ & $2(16.7 \%)$ & $14(40 \%)$ & $0.065+$ \\
\hline \multicolumn{6}{|c|}{ Medical risk factor } \\
\hline 1 & Present & $15(65.2 \%)$ & $4(33.3 \%)$ & $19(54.3 \%)$ & \\
\hline \multirow[t]{2}{*}{2} & Nil & $8(34.7 \%)$ & $8(66.7 \%)$ & $16(55.7 \%)$ & \\
\hline & Inference & \multicolumn{4}{|c|}{ Incidence of medical risk factors are more associated with ERCP with $\mathrm{p}=0.106$} \\
\hline
\end{tabular}


Table no 2: clinical symptoms and risk factors of patients studied.

Jaundice was presented in 18 of the 35 patients (51.4\%). Pain was a complaint in 32 patients (91.4\%). Jaundice in the past was elicited in $14(40 \%)$ of the patients, of these 4(33.33\%) were those without clinically apparent jaundice at presentation. 6 patients had history of intervention for biliary tract pathology. 4 (12.9\%) of whom had underwent cholecystectomy and 2(6.45\%) had undergone ERCP. 10 (32.25\%) patients were diabetics and 7(22.58\%) patients had hypertension. Three $(9.67 \%)$ patients had both hypertension and diabetes. Two patients were known case of ischemic heart disease.(Table 2)

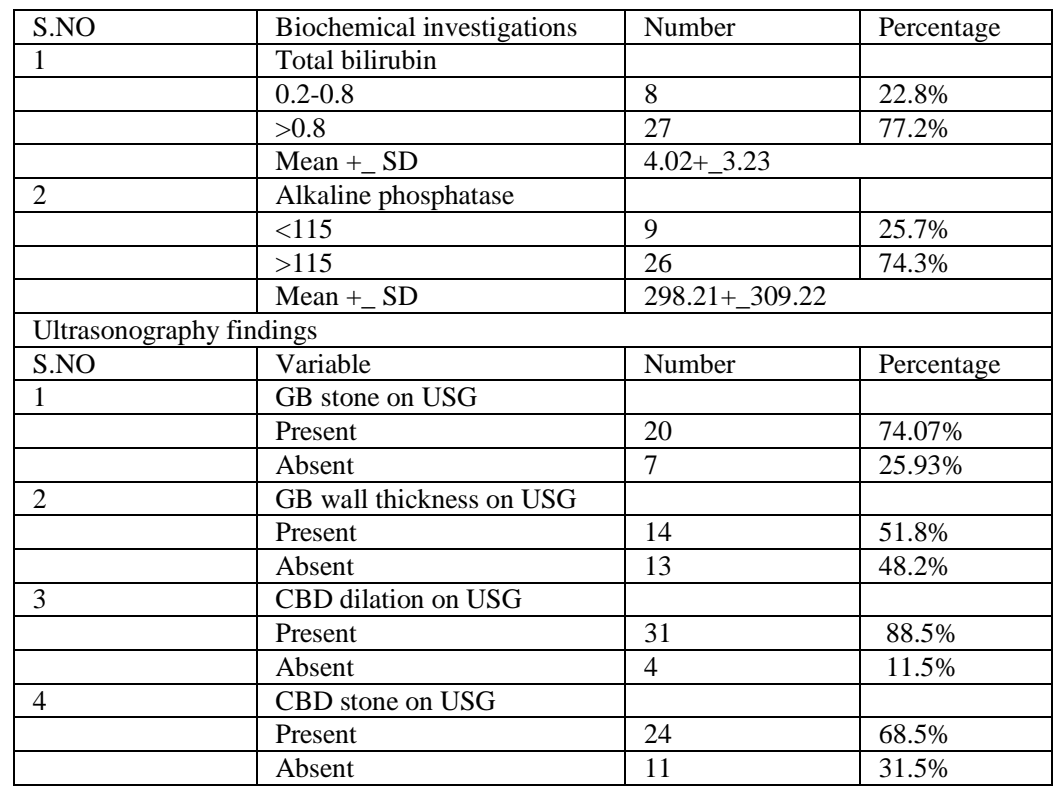

Table no 3: Biochemical and ultrasound findings in study.

Serum bilirubin was raised in 25 patients $(80.64 \%)$ with a maximum of $15.2 \mathrm{mg} / \mathrm{Dl}$ and a mean of 4.02 $\mathrm{mg} / \mathrm{dL}$ (SD 3.76). 24 (77.41\%) patients had a raised serum alkaline phosphatase (>115 IU/L) with a mean of 295.23 IU/L (SD 310.22). (Table 3) Ultrasonography has a sensitivity of $70.96 \%$ in detecting CBD calculi. Ultrasonography has a higher sensitivity of $90.32 \%$ in detecting ductal dilatation, an indirect evidence of CBD calculi

\begin{tabular}{|l|l|l|l|}
\hline S.NO & Procedure & Number & Percentage \\
\hline 1 & CBD exploration with cholecystectomy & 10 & $83.33 \%$ \\
\hline 2 & CBD exploration with choledocho-jejunostomy & 1 & $8.33 \%$ \\
\hline 3 & CBD exploration with transduodenal sphincteroplasty & 1 & $8.33 \%$ \\
\hline
\end{tabular}

\section{Table no-4: Surgeries performed in the study}

Twelve (38.70\%) patients underwent open surgical procedures. Included in this 12 are the 3 failed ERCP cases. Ten $(83.33 \%)$ patients underwent open CBD exploration with cholecystectomy. Nine (90\%) of these patients had a T-tube inserted into the CBD. One $(8.33 \%)$ patient who was cholecystectomized underwent choledocho-jejunostomy with jejuno-jejunostomy for a massively dilated proximal CBD $(3.5 \mathrm{~cm})$ with a narrowed distal CBD which had multiple impacted stones. One (8.33\%) patient underwent CBD exploration with transduodenal sphincteroplasty. This was done for an impacted stone at the ampulla of Vater. Four patients $(33.33 \%)$ in whom there were doubtful clearance of CBD calculi underwent an intraoperative cholangiogram. Stones were palpable intra-operatively in $10(83.33 \%)$ patients. Macroscopic evaluation revealed that there was a high incidence of pigment and mixed stones (Table 4).

\begin{tabular}{|l|l|l|l|}
\hline S.NO & Complication & Surgery & ERCP \& ES \\
\hline 1 & Present & $5(41.7 \%)$ & $2(8.7 \%)$ \\
\hline 2 & Absent & $7(58.3 \%)$ & $21(91.3 \%)$ \\
\hline 3 & Wound infection & 2 & 0 \\
\hline 4 & Pneumonia & 1 & 1 \\
\hline 5 & Acute renal failure & 0 & 1 \\
\hline 6 & Bile leak & 1 & 0 \\
\hline 7 & Hemorrhage & 1 & 0 \\
\hline
\end{tabular}




\section{Table no 5: Complications}

Bile culture and sensitivity of the aspirated bile was done in the patients who underwent open surgery. Positive cultures were obtained only in four patients. Escherichia coli, and Klebsiella spp were the most common organisms isolated from the bile cultures. Two patients who underwent CBD exploration developed wound infection and 1 developed pneumonia. Persistent bile leak was present in 1 patient who necessitated reexploration and closure of the leaking CBD rent. In patients who underwent ERCP with ES, 1 patient developed acute renal failure requiring dialysis and 1 developed pneumonia.

\begin{tabular}{|l|l|l|l|l|}
\hline S.NO & $\begin{array}{l}\text { Duration of hospital } \\
\text { stay(in days) }\end{array}$ & $\begin{array}{l}\text { ERCP+ } \\
\mathrm{n}=23\end{array}$ & Open surgery $\mathrm{n}=12$ & All patients $\mathrm{n}=35$ \\
\hline 1 & Min-max & $3-24$ & $18-42$ & $3-42$ \\
\hline 2 & Mean $+\_$SD & $11.84+66.01$ & $26.67+66.17$ & $17.58+\_9.46$ \\
\hline 3 & Inference & $\begin{array}{l}\text { Mean duration of hospital stay is significantly more associated with } \\
\text { open surgery with } \mathrm{p}<0.001\end{array}$ \\
\hline
\end{tabular}

Table no 6: Duration of hospital stay.

In patients who underwent ERCP alone, the mean hospital stay was 8.7 days (SD 4.08), ranging from 4 to 18 days. The patients who underwent cholecystectomy following ERCP had a mean hospital stay of 15.33 days (SD 6.042) ranging from 3 to 24 days. Overall, the patients in the ERCP group had a mean hospital stay of 11.84 days (SD 6.01). Patients who underwent open surgery had more prolonged hospital stay with a mean of 26.67 days (SD 6.17) ranging from 18 to 42 days. Comparing the endoscopy group and the open surgery group, the difference is significant $p<0.001$. (Table 6)

\section{Discussion}

Incidences of Choledocholithiasis have been found to be slightly higher in males compared with females in our study (1:0.93). Soon et $\mathrm{al}^{7}$ reported an incidence of 1.3: 1 . However, many other studies have shown an increased incidence in females. Way et al reported a female: male ratio of 3:1. ${ }^{8}$ Studies by Kumar et al have shown it to be $2: 1$ to $1: 1^{9}$. The reasons for the higher incidence in males could be the larger diameter cystic ducts which allow the passage of stones more easily. In our study, the most common age group affected is between 61-70 years. The mean age of the patients is 55.9 years. The incidence and prevalence of Choledocholithiasis trend to increase with age. Nathanson et al have reported the median age affected to be 59.6 years ${ }^{10}$. Sgourakis reported the age range to be between 46-89 years ${ }^{11}$. Hermann has shown that the incidence that begins in childhood, progresses steadily with a sharp increase between 35-55 years and continuous to show a gradual increase after 55 years ${ }^{12}$. So the disease is essentially of the elderly.

Pain localized to the upper abdomen on the right side was the most common presenting symptom in our study (93.5\%) followed by Jaundice (54.8\%) and fever (38.7\%). Although jaundice can occur in the absence of stones, the degree of hyperbilirubinemia correlates well with stones. In the study by way and associates the mean bilirubin levels for patients without stones were $4.9 \mathrm{mg} / \mathrm{dL}$ and for those with stones it was $9.1 \mathrm{mg} / \mathrm{dL}$. They also observed that the patients with bilirubin levels $>7 \mathrm{mg} / \mathrm{dL}$, there was a high likely hood of the stones. Bosc et al have shown that the incidence of stones increases as the bilirubin levels rise ${ }^{13}$. The predictability of jaundice increases when accompanied by fever or pain. Girard et al have studied that the more reliable clinical indication of Choledocholithiasis was jaundice associated with chills and fever ${ }^{14}$. Seven patients $(22.58 \%)$ have the classical triad of cholangitis with a mean bilirubin concentration of $7.64 \mathrm{mg} / \mathrm{dL}$. These could be the patients with longstanding cholestasis and consequent infection. The sensitivity of cholangitis is $95-100 \%$ for CBD stones $^{13}$. As is evident from the above discussion the clinical findings are not sufficient to establish the presence of CBD stones; imaging techniques are required for a more accurate assessment.

A previous history of jaundice, presence of medical risk factors were the risk factors identified in our study. Neoptolemos et al in their analysis of the risk factors in Choledocholithiasis found that for patients who underwent surgery the risk factors include age, jaundice, medical risk factors and bilirubin among many variables ${ }^{14}$. Similarly for ES it was acute cholangitis. The role of ultrasonography in detection of bile duct stone is less certain. The sensitivity deteriaotes as one goes from the proximal to distal duct. The absence of surrounded bile makes the stone difficult to differentiate from the peri ductal structures ${ }^{15}$. However, the sensitivity of ultrasonography in detecting ductal dilatation which is indirect evidence of CBD stones is very high. Hunt et al have showed that the probability of CBD stones increases as the duct size becomes large ${ }^{16}$. Laing et al while evaluating 53 patients with obstructive jaundice got a sensitivity of $29 \%$, specificity of $91 \%$ and an accuracy of $55 \%^{17}$. In Cronan's study a sensitivity of $67 \%$ was obtained for the ductal dilatation but for stones it varied from the proximal duct with a sensitivity of $58 \%$ to $23 \%$ for stones in distal duct ${ }^{18}$. Once stones are detected in ultrasound it can provide all information necessary for the management. In our study, sensitivity for dilatation and stones was $90.3 \%$ and $70.9 \%$ respectively.

In the era of invasive surgery, very few patients opt for open surgery. However, in the past, most CBD stones 
found at surgery were managed surgically with a minority managed by alternatively, namely, endoscopic retrograde cholangiopancreatography with or without endoscopic Sphincterotomy $(\mathrm{ERCP} / \mathrm{ES})^{19}$. Initial studies suggested that surgical CBD stone extraction is the recommended option for routine cases ${ }^{14}$. However, with more and more surgeons becoming familiar with the techniques of endoscopy, more number of patients is subjected to this procedure. In our study, ERCP/ES, was primarily used as a therapeutic modality and has a successful rate of $86.36 \%$. Lamber et al reported a success rate of $91.5 \%{ }^{135}$ and in the study by bose it was $74 \% .^{13}$

The need for subsequent cholecystectomy following ERCP/ES has been a controversial subject. Schreurs and colleagues concluded that when common bile duct stones are treated successfully by endoscopic Sphincterotomy and patients are free of symptoms, there is no need for routine prophylactic cholecystectomy. ${ }^{20}$ Soon et al were of the opinion that elective cholecystectomy is not warranted in patients with bile duct stones when the common duct can be cleared of stones by endoscopic Sphincterotomy. ${ }^{7}$ Any clinical predictors of further symptoms or complications arising from the retained gallbladder were not found in their study. In our study, in $52.6 \%$ of patients, ERCP with ES was the sole procedure performed. Five of the 7 patients $(71.4 \%)$ with cholangitis in our study were treated by the endoscopic method. it thus can be concluded that in patients with cholangitis endoscopic methods are the procedure of the choice.

However, Martin DJ Vernon DR., Toouli J concluded that pre or postoperative ERCP for bile duct clearance offers no apparent advantage over surgical exploration and also that ERCP necessitates increased number of procedures per patient. ${ }^{21}$

In the era of laparoscopic bile duct exploration, open surgery still gives excellent results as evident by the complete clearance of ductal stones in the 12 cases $(100 \%)$ that underwent open common bile duct surgery in our study. Mortality rates in patients undergoing surgery for CBD calculi ranges from $1 \%$ in relatively fit younger patients to $28 \%$ in the unfit and the elderly.138. It increases to $12-14 \%$ in younger patients undergoing surgery for cholangitis ${ }^{22}$. Moreover the mortality/morbidity is increased if a drainage procedure is included ${ }^{23}$. Morbidity/mortality for ERCP/ES ranges between 0.8 to $1.5 \%$ according to various reports ${ }^{22}$, the complications being hemorrhage, pancreatitis, cholangitis and perforation. Neoptolemos et al, in a randomized study reported a complication rate in the patients of the endoscopic group as $16.4 \% .^{24}$

In our study, open surgery or endoscopy did not produce any deaths. Seven (22.5\%) patients developed complications. Five $(41.6 \%)$ of these had undergone open surgery. This could be because much of the study population was elderly. In patients in whom endoscopy was the principal mode of treatment, 2 patients (10.5\%) developed complications. Incidence of complications were significantly associated with open surgery $(\mathrm{p}=0.078)$. Comparing the complication rates in these two categories is scientifically inaccurate as very different groups of patients are treated by these modes of therapy. This was the concept of 'apples and oranges' as stated by Cotton $^{25}$.Neoptelomos et al have stated that preoperative endoscopic Sphincterotomy, increases the chances of bile infection and thus leading to postoperative morbidity. They reported that their study did not show any significant difference in the complication rates between pre cholecystectomy ES patients and those treated by surgery alone ${ }^{24}$.

The differences in the hospital stay between the patients who underwent treatment by endoscopic methods and those who underwent open surgery was significant in our study ( $<<0.001)$. In a study comparing endoscopic methods and open surgery by Stephen et al the medical hospital stay was 5 days in the endoscopy group and 7 days in the open surgery group. ${ }^{26}$

\section{Conclusion}

ERCP with ES can be used as the only intervention for the treatment of CBD calculi in patients with a normal gall bladder. Cholecystectomy after ERCP and ES needs to be done only in patients with gall bladder pathology like stones and cholecystitis. Open CBD exploration is suitable for patients with large stones and in whom endoscopic retrieval is a failure. Intraoperative cholangiogram needs to be done only when there was doubtful clearance of CBD calculi. Complications tend to be more in patients who undergo open surgery. There is a significant decreased mean hospital stay in patients who undergo endoscopic treatment for bile duct calculi.

\section{References}

[1]. Cushieri A. Disorder of the biliary tract. In: Cushieri A, Steele RJC, Moosa AR, eds. Essential surgical practice, 4th ed. London: Butterworth Heinemann; 2002. P.375-454.

[2]. Cranley B, Logan H. Exploration of the common bile duct - the relevance of the clinical picture and importance of preoperative cholangiography. Br J Surg 1980; 67:869-72.

[3]. Bartlett MK, Waddell WR. Indication for common duct exploration; evaluation in 1000 cases. N Eng J Med, 1958; 258: 164-167.

[4]. DenBesten L, Doty JE. Pathogenesis and management of choledocholithiasis. In: Symposium in biliary tract disease. Surg Clin N Am 1981; 61 (4): 893-907.

[5]. Neoptolemos JP, Shaw DE et al, A multivariate analysis of preoperative risk factors in patients with Common Bile Duct Stone, Implications for treatment, Ann Surg, 1989; 209; 157-161.

[6]. Huang ZQ. Present status of biliary surgery in china. World J Gastroenterol 1998; 4 (Suppl 2): 8-9 
[7]. Soon KK, Byung SL et al. Is Cholecystectomy Necessary after ERCP for Bile Duct Stones in Patients with Gallbladder in situ? Korean J Intern Med. 2001; 16(4):254-259.

[8]. Way LW, Admirand WH et al. Management of Choledocholithiasis. Ann Surg 1972; 176: 347-359.

[9]. Kumar D, Garg PK, Tandon RK. Clinical and biochemical comparative study of different types of common bile duct stones. Indian J Gastroenterol 2001; 20: 187-190.

[10]. Nathanson LK, O'Rourke NA et al. Postoperative ERCP versus laparoscopic choledochotomy for clearance of selected bile duct calculi: A randomized trial. Ann of Surgery 2005; 242(2): 188-92.

[11]. Sgourakis G, Karahotas K. Laparoscopic common bile duct exploration and cholecystectomy versus endoscopic stone extraction and laparoscopic cholecystectomy for choledocholithiasis. A prospective randomized study.

[12]. Hermann RE. The spectrum of biliary stone disease. Am J Surg 1959: 158: LY 171-173.

[13]. Bose SM. Predictors of Choledocholithiasis. Ind J Surg 2002; 64: 335-338.

[14]. Neoptolemos JP, Shaw DE, Can-Locke DL. A multivariate analysis of preoperative risk factors in patients with common bile duct stones, Implications for treatment, Annals of Surgery 1989; 209(2): 157-61.

[15]. Gross BH et al, Ultrasonic evaluation of Common Bile Duct stones: Prospective comparison with Endoscopic Retrograde Cholangiopancreatography, Radiology

[16]. 1983; 146: 471- 474 .

[17]. Hunt DR, Reiter L, Scott A]. Preoperative ultrasound measurement of bile duct diameter: Basis of selective cholangiography. Aust NZ J Surg 1990; 60: 189-192.

[18]. Laing FC et al. Choledocholithiasis and Cystic Duct obstruction: Difficult ultrasonographic diagnosis, Radiology 1983; 146: 475479.

[19]. Cronan II, US diagnosis of choledocholithiasis: A reappraisal, Radiology 1986; 161: 133-134.

[20]. Fletcher DR Laparoscopic cholecystectomy: Role of pre-operative and post-operative endoscopic retrograde cholangiopancreatography and endoscopic sphincterotomy. Gastrointest Endosc Clin N Am 1993; 3: 249-259.

[21]. Schreurs WH, Vles WJ, Stuifbergen WH, Oostvogel HJ. Endoscopic management of common bile duct stones leaving the gallbladder in situ. A cohort study with long-term follow-up. Dig Surg 2004; 21(1): 60-4.

[22]. Martin Di, Vernon DR, Toouli J. Surgical versus endoscopic treatment of bile duct stones. Cochrane Database of Systematic Reviews 2006, Issue 2. Art. No.: CD003327. DOI: 10.1002/14651858.CD003327.pub2.

[23]. Somnay K, Carr-Locke DL. Stones in the bile duct: endoscopic apprpoaches. In: Blumgart LH et al (editors). Surgery of the liver and biliary tract, 3th ed. Churchill Livingstone 2000 1(4): 749.

[24]. Capper WM. External cholecloclioduodenostomy, an evaluation of 125 cases. Br J Surg. 1961; 49: 292-300.

[25]. Neoptolemos JP, Carr LD et al. Prospective randomized study of preoperative endoscopic sphincterotomy versus surgery alone for common bile duct stones. Br Med J. 1987; 294: 470.

[26]. Cotton PB. Endoscopic management of bile duct stones (apples and oranges). Gut 1984; 25: 587-597.

[27]. Stephen CS, Hartley C. Choledocholithiasis: Endoscopic Sphincterotomy or Common Bile Duct Exploration. Ann Surg. June 1991; 627-633. 